\title{
Modulating Optical Signal to Improve the Images by Using a Self Crossed Diffraction Grating
}

\author{
N. I. Hendawy \\ (Department of Physics, Faculty of Science, Benha University, Qalubia, Egypt)
}

\begin{abstract}
A method for recording good images on high resolutionphotographic plate and their retrieval was described.This method depend on the modulating optical signal by a crossed diffraction grating. Two symmetrical first and second orders werefiltered to modulate the real amplitude object.These ordersrepresent the optical modulated signalson the photographic plateby a given frequency of crossed diffraction grating and another frequency from itself crossed diffraction grating respectively. Different irradiance distributions for a different modulated signals were appeared. Improved images were obtained from a given modulating signal. The irradiance distributions and visibilitywere calculated theoretically and experimentally.
\end{abstract}

Keywords: crosseddiffractiongrating, irradiance distribution, modulated image, optical signal, spatial carrier.

\section{Introduction}

In the age of information and images, scientists have been working all over the world on the use and development of different ways to develop and improve the quality of information, as well as, storage. These different methods can be electrical, electronic and optical. There are several techniques for optical image processing. All techniques depend on modulating the optical signals with spatial carriers. If the object has a phase information, reference beam must be introduce to interferes with the signal beam. The high- frequency fringe system was formed on a photographic plate [1-2]. If the object has only real amplitude variations, the amplitude diffuser [3-7] or amplitude diffraction grating [8-15] was used to modulate the signal to be stored. Due to increase the capacity of the stored images on the photographic plate, signal to noise ratio (SNR) as a result of the orientation of the cross-talk and the visibility of the retrieved images were studied theoretically and experimentally[4]. Mathematical expressions for the irradiance distribution and visibility distribution in the neighborhood of the geometrical image plane of a crossed grating were derived [9]. According to Abbe's theory[10], it is known that an image requires two contributions: undiffracted light (zeroth order) which provides the overall illumination and the diffracted light (higher order maxima) which provide the transfer of information.Noting that the zero-order maximum alone does not produce an image. If more higher orders could be admitted and contribute to image formation, the image would contain more information, more details. Carrier- frequency photography with incoherent light can be employed for storing a number of real amplitude objects (signals) on a single photographic plate. The signal to be stored was placed, successively in contact with a crossed diffraction grating (grid) and this ensemble was imaged on a photographic plate. The frequency- plane mask selected two different spectral orders for each object. Different intensity distributions modulate different fringe systems in the image plane and could be retrieved with appropriate filters. The grid remained stationary throughout the operation of storage [11]. A crossed grating with spatial frequency (40lines $/ \mathrm{mm}$ ) could be used to store up to eight images [12]. In this article, we describe and develop a method to improve the quality of the image of the real amplitude object by another optical signals from the self grating. Determine the irradiance distributions of the optical modulated signals and visibility theoretically and experimentally.

\section{Experimental Set-Up}

To record a real amplitude object on a photographic plate, a crossed grating was used as a carrier frequency to modulate the real amplitude object. Fig.1 represents the set-up for recording one or more real amplitude objects on a single photographic plate. The object was placed in contact with a crossed grating (grid) in plane $\left(\mathrm{x}_{1}, \mathrm{y}_{1}\right)$. The ensemble was illuminated by a parallel beam monochromatic light. A large number of diffracted images were formed in the frequency plane $\left(\mathrm{x}_{2}, \mathrm{y}_{2}\right)$ as in fig. 2 . A high pass spatial filter with two holes was placed in the frequency plane $\left(\mathrm{x}_{2}, \mathrm{y}_{2}\right)$. It passed only two symmetrical diffracted orders. The lenses $\mathrm{O}_{1}$ and $\mathrm{O}_{2}$ (has the same focal length) formed the image of the object and grid on the photographic plate in plane $\left(\mathrm{x}_{3}, \mathrm{y}_{3}\right)$. In this case, the image have a unit magnification. In practice, the lenses $\mathrm{O}_{1}$ and $\mathrm{O}_{2}$ may be replaced by a single lens, then, the image may have any magnification [12].

To retrieve the stored images, remove the ensemble (object and grid) and put the developed photographic plate in the same plane $\left(\mathrm{x}_{1}, \mathrm{y}_{1}\right)$. The spectrum of the modulated image was appeared in the plane $\left(\mathrm{x}_{2}, \mathrm{y}_{2}\right)$.Isolated the zeroth order (direct image) by a filter with a circular apertures. The image of the real amplitude object wasappeared in plane $\left(\mathrm{x}_{3}, \mathrm{y}_{3}\right)$. 


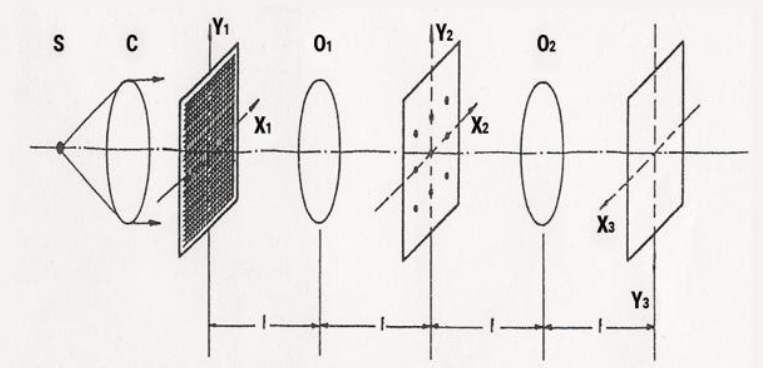

Fig.1 Experimental set-up for recording the modulating signals and its retrieval.

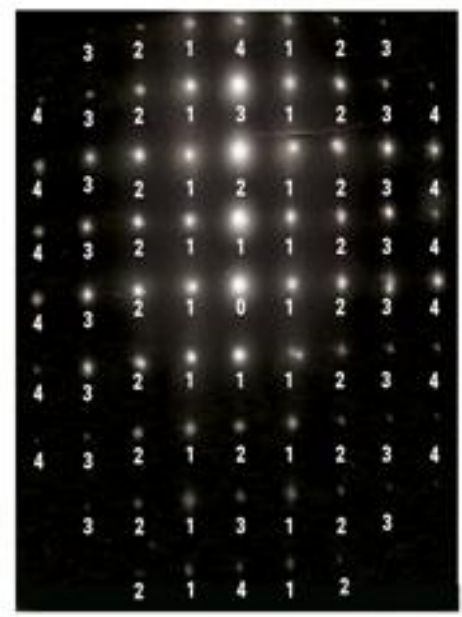

Fig.2 Image of the optical spatial carriers due to a crossed grating.

\section{Results And Discussions}

In our method, a monochromatic light with a wave length $(\lambda=632.8 \mathrm{~nm})$ and a crossed diffraction grating with frequency (100 lines/ $\mathrm{mm}$ ) were used. A crossed diffraction grating was used to record the modulating optical signal by filtering the two symmetrical first orders. If the frequency of the crossed diffraction grating with(50 lines/ $\mathrm{mm})$ was used, another optical modulating signals can be recorded on the same photographic plate. This a crossed diffraction grating not used, but the two symmetrical second orders were filtered from the same used crossed diffraction grating $(100$ lines/ mm). Figs. 3a, 3bshowed image of the spectrumof modulating optical signals (carriers) in the plane $\left(\mathrm{x}_{2}, \mathrm{y}_{2}\right)$ in case of the retrieved images. These represent the frequency grating 100 lines / $\mathrm{mm}$ and 50 lines / $\mathrm{mm}$ respectively. It is clear that the frequency of optical modulating signals as in fig.3a equal to twice the frequency of optical modulating signals as in fig. $3 \mathrm{~b}$. Fig. $3 \mathrm{c}$ shows algebraic summation of the irradiance distributions from fig. 3a and fig. 3b respectively. The irradiance distribution at the secondspectrum of optical modulating signal is greater than the first spectrum of optical modulating signal. Visibility of the secondspectrum of optical modulating signal is greater than the firstspectrum of optical modulating signalas in fig. $3 \mathrm{c}$. The quality of retrieved images which corresponding to $1^{\text {st }}, 2^{\text {nd }}$ and $\left(1^{\text {st }}+2^{\text {nd }}\right)$ filtered were appeared in the plane $\left(x_{3}, y_{3}\right)$ as shown in fig. 4.

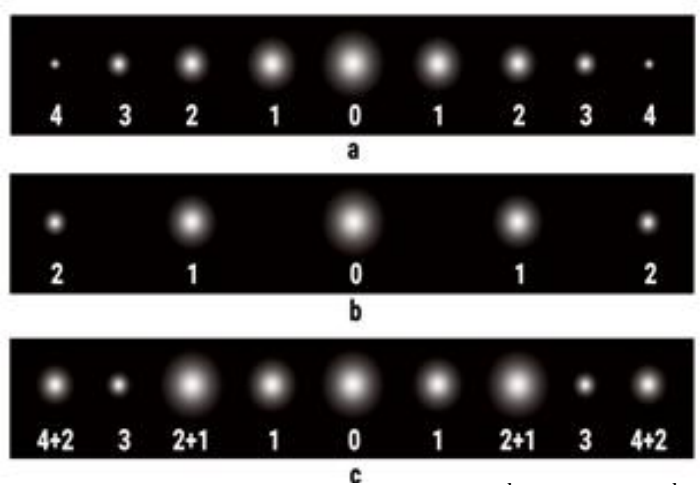

Fig.3 Images of the optical modulating signals due to (a) $1^{\text {st }}$ (b) $2^{\text {nd }}$ (c) $1^{\text {st }}+2^{\text {nd }}$ were filtered from the a crossed grating. 


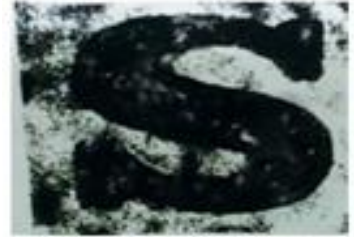

a

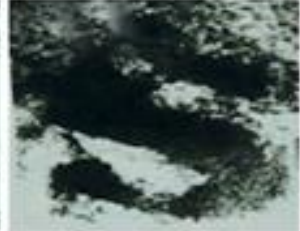

b

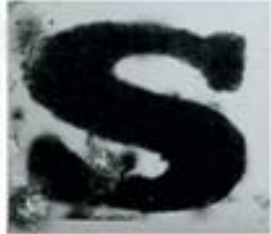

c

Fig.4 Retrieved images of the real amplitude objects due to (a) $1^{\text {st }}$ (b) $2^{\text {nd }}$ (c) $1^{\text {st }}+2^{\text {nd }}$ were filtered from the a crossed grating.

The intensity distribution I for Fraunhofer pattern of a diffraction grating due to $\mathrm{N}$ slitsis given by [18]:

$\mathrm{I}=\mathrm{I}_{0} \mathrm{~N}^{2}[\sin (\alpha) / \alpha]^{2}(1)$

provided that, $\alpha=(\mathrm{ka} / 2) \sin (\theta)$, where $\mathrm{k}$ is the wave number, a is the width of slit of the grating and $\theta$ gives the direction of the diffracted orders, $\mathrm{N}$ is the frequency of diffraction grating and $\mathrm{I}_{0}$ is the intensity at $\theta=0$ direction. The value of $(\sin \alpha / \alpha) 2$ equal to 0.047 and 0.017 for $\alpha$ equal to $1.43 \pi$ and $2.46 \pi$ respectively[19].The total intensity distributions $I_{T}$ for the spectrum modulated images when $\left(1^{\text {st }}+2^{\text {nd }}\right)$ were filtered in fig. $3 \mathrm{c}$ is given by:

$\mathrm{I}_{\mathrm{T}}=\mathrm{I}_{2}+\mathrm{I}_{1}=\left\{\left[\mathrm{I}_{0} \mathrm{~N}^{2}(\sin \alpha / \alpha)^{2}\right]\right\}_{2}+\left\{\left[\mathrm{I}_{0} \mathrm{~N}^{2}(\sin \alpha / \alpha)^{2}\right]_{1}\right\}=\mathrm{I}_{0} \mathrm{~N}^{2}\left\{\left[(\sin \alpha / \alpha)^{2}\right]_{2}+\left[(\sin \alpha / \alpha)^{2}\right]_{1}\right\}$

where $I_{2}$ and $I_{1}$ represent the intensity distributions at the second and first spectrum of modulated images as shown in fig. 3a and fig. 3b. In our experiment, $\mathrm{I}_{0}=10^{-3} \mathrm{mw} / \mathrm{mm}^{2}, \mathrm{~N}^{2}=10^{4}$ lines $/ \mathrm{mm}^{2}\left[(\sin \alpha / \alpha)^{2}\right]_{2}=0.047$ and $\left[(\sin \alpha / \alpha)^{2}\right]_{1}=0.017$. Then, The total intensity distributions $\mathrm{I}_{\mathrm{T}}$ for the spectrum modulated images occurred when $\left(1^{\text {st }}+2^{\text {nd }}\right)$ were filtered as in fig. $3 \mathrm{c}$. It equal to $0.64 \mathrm{mw} / \mathrm{mm}^{2}$. The visibility $\mathrm{V}=0.9$ at minimumintensity distribution equal to $0.03 \mathrm{mw} / \mathrm{mm}^{2}$.

\section{Conclusion}

The frequency ofspatial carriers of a crossed diffraction grating are used to modulate real amplitude objects. Different frequencies of spatial carriers can be taken from same a crossed grating. Different intensity distributions can be recorded on the same photographic plate. This is possible by filtering two successive orders. At a certainintensity distribution, good quality of retrieved image can be obtained. Highest irradiance distribution and visibility can be obtained when the two successive orders are taken from the crossed diffraction grating. This method can be used torecord multiple images without azimuth angle, addition and subtraction of images. The resultant signal to noise ratio is few.

\section{References}

[1] G Rakuljic and VectorLryva, Optical data storage by using orthogonal wave length-multiplexed volume holograms, Optic Letters, 17(20),1992, 1471-1473.

[2] G Nordin, Effects of crosstalk on fidelity in page - oriented volume holographic optical data storage, Optics Letters, 18(18), 1993, 1553-1555.

[3] C Grover, A new method of image multiplexing using a random diffuser, J.Opt.Soc.Am, 26, $1972 \mathrm{a}, 1071$.

[4] N Hendawy, Effect of cross-talk Phenomenon on the retrieved images, Egyptian J. Physics, 35(2), 2004, 1-13.

[5] A Nasser Moustafa and N.I.Hendawy, Comparative phase-shifting digital speckle pattern interferometry references beam technique, Egyptian J.Solids, 26(2), 2003, 225 -229.

[6] T EL-Dessouki, N.I.Hendawy and A.A.Zaki, Measuring birefringence of curved sheet and single-crystals by double-exposure speckle photography, Optics and Lasers in Engineering, 47(6), 2009, 622-628.

[7] T EL-Dessouki, M.Roushdy, N.I.Hendawy, M.M.Naoum, A.A.Zaki, Optical measurments of thermotropic liquid crystals, J.Modern Physics, 4, 2013, 39-48.

[8] S Mallick, Interferometry with an achromatic-fringe system, Optica Acta, 19(9), 1972, 739-747.

[9] R Grousson and S.Mallick, Effect of source size and spectral width on the irradiance distribution in the defocused image of a grating, Optica Acta, 22(12), 1975, 981-987.

[10] J.R. Meyer and Arendt, Introduction to Classical and Modern Physics, Third Edition (Prentice - Hall Tnternational Edition, 1989, 33).

[11] S Mallick, Multiple Image Storage Using a Crossed Grating, Optics Communication, 7(4), 1973,427.

[12] R Grousson and S.Mallick, Multiple image storage using a crossed grating, Nouvelle Revue d'Optique, 7(2), $1976,77$.

[13] R Grousson and R.S.Kinany, Multicolor image storage on black and white film using a crossed grating, J.optics, 9(6), 1978, 399.

[14] P Vincent, A finite-difference method for dielectric and conducting crossed gratings, Optics Communications, 26(3), 1978, 293296.

[15] R Grousson and S.Mallikc, White light image processing with Linbo3, Applied Optics, 19(11), 1980, $1762-1767$.

[16] R Grousson and S.Mallick, Image contrast reversal with a photorefractive recording medium, Applied Optics, 20(9), 1981, 17191723 .

[17] S Abd EL-Gahany and N.I.Hendawy, Effect of the spectral profile shape and the spatial frequency on the visibility in thedefocused image of the grating, Egyptian J.Solids, 33(3), 2004, 9-22.

[18] N. Subrahmanyam and B. Lal, A Text Book of Optics, Seventh Edition (S. Chand and Co. Pvt Ltd: Ram Nagar, New delhi , 1976, 364).

[19] E.Hecht, Optics,fourth edition (Academic press: New York, 2004, 452-463) 